\title{
АУТОИММУННЫЙ ПОЛИГЛАНДУЛЯРНЫЙ СИНДРОМ 1 ТИПА - КЛИНИЧЕСКИЙ ПОЛИМОРФИЗМ И ОСОБЕННОСТИ ТЕЧЕНИЯ
}

\author{
${ }^{1}$ Логачев М.Ф., ${ }^{1}$ Сичинава И.Г., ${ }^{2}$ Карманов М.Е.,

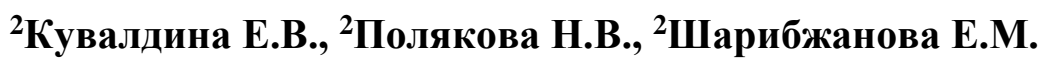 \\ ${ }^{1}$ ФГАОУ ВО РНИМУ им. Н.И.Пирогова Минздрава России \\ ²ОСП РДКБ ФГАОУ ВО РНИМУ им. Н.И.Пирогова Минздрава России, \\ Москва, Россия
}

Аутоиммунный полигландулярный синдром (АПС-1) - редкая наследственная аутоиммунная болезнь, которая встречается с частотой 1:100000. В настоящее время достигнуты определенные успехи в диагностике АПС-1, но в то же время клинический полиморфизм и многообразие неспецифических симптомов, предшествующих манифестации основных клинических проявлений АПС-1 - хронического кожно-слизистого кандидоза (ХКСК), гипопаратиреоза (ГПТ) и первичной хронической надпочечниковой недостаточности (ХHН) - нередко является причиной запоздалой диагностики этой болезни и представляет угрозу для жизни и здоровья пациентов.

С целью оптимизации клинико-лабораторного скрининга и ранней диагностики АПС-1 нами были проанализированы истории болезни 39 пациентов в возрасте от 4 лет 3 мес- до 17 лет 11 мес (девочки-25, мальчики-14), находившихся с 2005 по 2021 годы под наблюдением в РДКБ, где неоднократно проходили стационарное обследование и лечение. Изучены варианты манифестации, клинические компоненты и особенности течения, сочетание эндокринных и неэндокринных проявлений болезни.

Манифестация первых проявлений АПС-1 приходилась на широкий возрастной диапазон- от 1 мес до 13 лет. При этом следует отметить, что если дебют ХКСК приходился на 0-6 лет жизни, то для ГПТ характерен возрастной период - от 8 мес до 12,6 лет, а для ХНН - 4-10,7 лет. Анализ последовательности манифестации клинических проявлений болезни выявил определенные закономерности. В 51,6\% наблюдений первым проявлением болезни был ГПТ, в 35,5\% - ХКСК, а у $12,9 \%$ пациентов - ХНН. В большинстве наблюдений (64,5\%) у одного и того же пациента наблюдалось сочетание 3 основных компонентов АПС-1, у 8 - установлено наличие двух компонентов, и только у 2 - один основной компонент заболевания. Среди других клинических проявлений АПС-1 имели место аутоиммунный тиреоидит, аллопеция, аутоиммунный гепатит, аутоиммунная энтеропатия, гипергонадотропный гипогонадизм, СД1, а среди «неосновных» гипоплазия зубной эмали, ониходистрофия и В12-дефицитная анемия.

Окончательный диагноз АПС-1 подтверждался на основании генетического исследования. Минимальный возраст пациента на момент установления диагноза составлял 2 года, максимальный 15 лет. Длительность от манифестации первых симптомов болезни до постановки диагноза в большинстве наблюдений (67,7\%) составляла от 2 до 5 лет, а у 6,5\% пациентов - 5-10 лет и только у $25,8 \%$ пациентов диагноз был установлен в течение года с момента появления первых симптомов болезни. Стаж болезни на момент последней госпитализации варьировал от 1,8 до 17,7 лет, в большинстве наблюдений (64,7\%) составляя 10 и более лет.

Таким образом, для пациентов с АПС-1 характерно начало болезни с неспецифическими проявлениями и длительным латентным периодом до выраженной манифестации симптомов. Учитывая, что в дебюте болезни пациенты нередко вначале попадают не к эндокринологу, требуется настороженность со стороны врачей смежных специальностей в отношении ранних проявлений АПС1 у детей. Нами были оптимизированы алгоритмы клинического и лабораторного скрининга АПС-1, что способствовало более ранней постановке диагноза, своевременному началу терапии и улучшению исходов для пациентов. 